\section{Urologia \\ Internationalis}

\section{Evangelos N. Liatsikos ${ }^{a}$ Kostantinos Assimakopoulos ${ }^{a}$ Jens-Uwe Stolzenburg ${ }^{\text {b }}$}

a Department of Urology, University Hospital of Patras, Patras, Greece; ${ }^{b}$ Department of Urology, University of Leipzig, Leipzig, Germany
Urol Int 2008;80:226-230

DOI: $10.1159 / 000127331$

\title{
Quality of Life after Radical Prostatectomy
}

Key Words

Radical prostatectomy $\cdot$ Laparoscopy $\cdot$ Quality of life

\section{Abstract}

Introduction: Radical prostatectomy is a standard approach to the management of prostate cancer. As the oncological outcome has improved, focus has drawn to the postoperative amelioration of health-related quality of life (HRQOL). Materials and Methods: The instruments that have already been used, or may be used, in assessing the HRQOL postoperatively in men with prostate cancer are presented. Results: Urinary and sexual dysfunction as well as fecal incontinence form the three possible major complications after surgery which affect the patient's quality of life. Meticulous counseling regarding the potential morbidity associated with the operation that may affect the patient's quality of life should be made before the patient gives his informed consent. Conclusions: Questionnaires only partially help to identify patients with dysfunction, especially sexual and bowel dysfunction, and careful patient counseling with their partners present is the best way to evaluate the presence of dysfunction pre- or postoperatively.

Copyright $\odot 2008$ S. Karger AG, Base

\section{Introduction}

The incidence of prostate cancer is continually increasing in developed countries, reflecting the widespread use of prostate-specific antigen screening. The overall survival rate for men with prostate cancer in the United States is considered to be $97 \%$ at 5 years, $79 \%$ at 10 years and $57 \%$ at 15 years [1]. Different treatment approaches are described in published series, depending on the stage of the disease, age and patients' choice. As stated for every cancer treatment, the primary goal of treatment is a combination of the longest survival, least complications and ameliorated health-related quality of life (HRQOL). In this concept, radical prostatectomy (RP) currently reflects the state-of-the-art in the management of clinically localized prostate cancer. The procedure can be performed by the perineal, retropubic or laparoscopic approach.

Laparoscopic (transperitoneal) RP (LRP) and endoscopic (extraperitoneal) RP have been established as safe and effective therapeutic approaches [2-6]. In 1999, Guillonneau and Vallancien [7] reported their experience with LRP in 65 men with prostate cancer and proposed that LRP could become a routine urologic procedure. Salomon et al. [8] stated that there is no significant difference with regard to oncological outcome after retropubic,

\section{KARGER \\ Fax +41613061234 E-Mail karger@karger.ch} www.karger.com
Jens-Uwe Stolzenburg, MD, PhD

Department of Urology, University of Leipzig

Liebigstrasse 20

DE-04103 Leipzig (Germany)

Tel. +49341971 7600, Fax +49341971 7609, E-Mail stolj@medizin.uni-leipzig.de 
perineal or laparoscopic RP. The quality of life after LRP depends primarily on urinary, bowel, and sexual function. Nerve-sparing approaches as well as techniques for bladder neck reconstruction and urethrovesical anastomosis have been developed in an effort to minimize implications after LRP and improve functional results [3].

As prostate cancer is increasingly diagnosed at early stages and technical refinements regarding LRP are developed, longer survival outcomes are accomplished. Localized prostate cancer is potentially curable with the appropriate surgical technique. However, postoperative complications, leading to dysfunction of the urogenital system, may last for a lifetime. Urologists are starting to take the postoperative HRQOL into account when considering the treatment management of prostate cancer or, at least, they should do so.

In this study, we review the current literature related to HRQOL after LRP, and present the instruments that have already been used, or may be used, in assessing the postoperative HRQOL in men with prostate cancer.

\section{Urinary Function}

Urinary incontinence (UI) occurs in 5-74\% depending on the surgical technique, the operative experience of the urologist, the definition of UI, and the method of evaluation [9]. It usually appears immediately after the operation but continence recovers partially or fully during the first months. Unfortunately UI will be a lifetime problem for some patients. The symptoms range from post-void dribbling to total loss of bladder control and may appear rarely or occasionally. Urinary dysfunction influences the patient's daily life, social relationships, sleep and of course self-respect.

Because of the major life restrictions that UI may induce, an instrument able to distinguish its presence and estimate its impact on the patient's daily life is needed. A comprehensive question naire, which is sensitive to changes over time, seems to be an efficient instrument. At present, there is no common consensus on which questionnaire should be considered as the standard or mandatory instrument for the follow-up of patients and for scientific debate. Furthermore most of the questionnaires used are not necessarily validated but are used in terms to demonstrate 'comparable results'. Various studies have been published, but further evaluation and mainly validation of the current questionnaires are indispensable. In the scientific debate, two instruments primarily developed to estimate general treatment outcomes have been consid- ered as feasible: the Functional Living Index-Cancer and the European Organization for the Research and Treatment of Cancer Quality of Life Questionnaire Version 2 (EORTC QLQ-C30) [10, 11]. Primarily, these two questionnaires were intended only for application on latestage prostate cancer and neither of them was designed to evaluate the specific impact of UI after prostatectomy. For these reasons, there has been an effort to develop more specific questionnaires such as the King's Health Questionnaire (KHQ), the Incontinence Quality of Life Questionnaire (I-QOL), the Bristol Female Lower Urinary Tract Symptoms Questionnaire, the York Incontinence Perceptions Scale (YIPS), and the Incontinence Impact Questionnaire (IIQ).

The KHQ has been validated in 285 women who underwent urodynamic studies, and it showed internal consistency, test-retest reliability, and criterion validity [12]. I-QOL is composed of 28 items and has been reported to have a negative correlation with the self-reported severity of incontinence, sorting of severity, and the frequency of medical appointments in 1 year [13]. Both the above instruments are reported to correlate with the Short Form 36 Health Survey (SF36). The Bristol Female Lower Urinary Tract Symptoms Questionnaire has not yet been studied to estimate quality of life but seems to correlate with frequency volume charts and perineal pad tests [14]. The YIPS evaluates the patient's psychosocial adjustment to chronic UI with regard to control, coping, and acceptance. A positive adjustment is found to correlate with a subject-reported daily reduction in UI episodes [15]. The IIQ was developed by the Continence Program for Women Research Group in Richmond and has become the most widely used instrument to date [16]. It includes 30 items and validates the impact of UI on the daily life of women. The IIQ correlates with subject-reported incontinence episodes and pad tests. The IIQ has been modified to a simplest form containing 7 items and has been renamed the IIQ-7 [17]. Yet neither of these instruments is widely used in men.

In 2005, Namiki et al. [18] evaluated the quality of life of 166 patients: 45 after LRP and 121 after retropubic RP (RRP). They used two questionnaires: the SF36, which is a general HRQOL questionnaire, and the disease-specific UCLA Prostate Cancer Index (UCLA-PCI). They showed a different pattern of changes with regard to HRQOL in the first year postoperatively. The LRP group had less body pain and physical limitations immediately after surgery as validated by SF36, but had a delayed recovery of urinary function compared to the RRP group. When dividing the LRP group into two subgroups ac- 
cording to surgical period, an improvement in HRQOL was observed in the most recently treated LRP subgroup. The same comparison was described by Roumeguere et al. [19], but these authors used a strict definition for continence which was the absence of any leakage and the use of any pad at any time postoperatively. They reported a similar continence rate between the two approaches but more rapid continence recovery after RRP [19].

Anastasiadis et al. [20] compared the functional results in men after LRP versus RRP. They used a questionnaire derived from the International Continence Society (ICS) male questionnaire in order to evaluate UI after the two surgical procedures. They described an earlier recovery of continence in the LRP group but a similar rate of continence 1 year postoperatively [20]. Rassweiler et al. [21] reported similar continence rates 1 year after either LRP (early or late) or open RP. Eden et al. [22] stated that $90 \%$ of their patients were pad-free 1 year after LRP.

Recently, using the disease-specific UCLA-PCI questionnaire, Namiki et al. [23] evaluated the quality of life after RP by the retropubic, laparoscopic or perineal approach. In this study, the LRP group seemed to show delayed recovery of urinary function, compared with the other two groups. The rate of bilateral nerve sparing in the LRP group was lower than in the RRP group, which partly explains the difference in the timing of continence recovery. When the same rate of nerve-sparing technique was used in each group of patients, the time to continence recovery was similar.

Various mechanisms have been reported in the literature to be responsible for post-prostatectomy UI. Preservation of the integrity of the pelvic floor and/or external urethral sphincter muscle as well as anterior urethral support seem to play an important role in postoperative continence. Most of the questionnaires mentioned above were developed in order to evaluate the presence and degree of incontinence in the female population. Unfortunately, these female scores have not been shown to provide reliable data in men with incontinence after RP.

\section{Sexual Function}

Sexual function enfolds sexual desire, penile ability for erection, achievement of orgasm, frequency of intercourse and other aspects. At the age of 50-80 years, sexual desire, erection capacity and orgasm pleasure decreases in $50-70 \%$ of men, causing enormous distress for them and their sexual partners [24]. Reports on sexual function in men after RP show large variability with re- gard to potency. Until the introduction of nerve-sparing concepts, almost all men showed no regain of sexual function after surgery. But even when the anatomical integrity of the nerves seems to have been preserved, various categories of factors have been demonstrated to affect the recovery of potency. Comorbidities such as diabetes, hypertension, arterial insufficiency or even the patient's age itself negatively influence erectile function. The recovery of sexual function more likely depends on the surgeon than on the treatment approach (retropubic, laparoscopic or perineal technique).

Due to the absence of a standardized methodology, the evaluation of erectile function seems to be difficult if not impossible. Because of the lack of a validated questionnaire, authors have used different instruments in order to estimate sexual function. None of these instruments was developed to assess the objective or physiologic measures of erectile function. Thus, their use is restricted as it is not clear if mental or physical parameters are responsible for the dysfunction observed.

The Cancer Rehabilitation Evaluation System (CARES), the EORTC QLQ-C30 (mentioned above), the International Index of Erectile Function (IIEF), the O'Leary Scale, the Prostate Cancer Treatment Outcome Questionnaire (PCTO-Q) and the Expanded Prostate Cancer Index Composite (EPIC) questionnaires are predominantly used in the assessment of sexual function in men with prostate cancer. CARES was developed by the Cancer Inventory of Problems and Situations to estimate rehabilitation needs and the quality of life of patients with prostate cancer $[25,26]$. The EORTC QLQ-C30 was introduced by the EORTC genitourinary group to assess both sexual function and urinary symptoms. The O'Leary Scale, named after its promoter, was designed to measure various domains of sexual function. O'Leary et al. [27] reviewed published reports and developed a short measure of sexual function in men before and after treatment for prostate cancer. The Prostate Cancer Treatment Outcome Questionnaire (PCTO-Q) was designed to estimate side effects and quality of life in patients treated for prostate cancer [28]. EPIC was developed at the University of Michigan and it is a validated instrument for the estimation of the quality of life in men after treated prostate cancer. It can be used to evaluate urinary, bowel, sexual and hormonal functions [29].

Wagner et al. [30] investigated the impact of nerve sparing during LRP on the sexual function of men using the EPIC questionnaire. The sexual function subscale score (SFSS) was reported as a percentage of baseline preoperative sexual function. In patients with unilateral 
nerve sparing, improvement in potency was reported between 3 and 6 months and showed a small change at 12 months. 35\% of these patients reported successful intercourse at 12 months. In contrast, potency continued to improve over time until 12 months after bilateral nerve sparing with $72 \%$ of these patients reporting successful intercourse. $57 \%$ of patients $\leq 58$ years old and $29 \%$ of patients $>58$ years old reported intercourse at 6 months postoperatively. The results increased to 74 and $41 \%$, respectively, at 12 months. The mean SFSS at 1 year was $61 \%$ of the baseline for the bilateral nerve-sparing group and $57 \%$ of the baseline for the unilateral nerve-sparing group.

Anastasiadis et al. [20] compared LRP with RRP with regard to the sexual function postoperatively. For this purpose, they used a questionnaire, derived from the ICS male questionnaire, in which potency was described by the ability to achieve and maintain an erection efficient for sexual intercourse. They showed no statistically significant difference in the potency rate 1 year postoperatively independent of the approach. The potency rate for the LRP group was $41 \% 1$ year after surgery. In the subgroup analysis of nerve preservation, potency rates increased in favor of the bilateral approach. For patients $<60$ years of age, the bilateral nerve-sparing technique led to $81 \%$ potency rate 1 year after LRP. Katz et al. [31] also stressed the significant role of bilateral nerve preservation in better sexual function postoperatively. $87.5 \%$ of patients with a bilateral nerve-sparing and $30 \%$ of men without a nerve-sparing approach had spontaneous erections 1 year postoperatively. Eden et al. [22] stated that $62 \%$ of men treated with bilateral nerve-sparing LRP had erections 12 months after the operation.

Roumeguere et al. [19] compared sexual function in patients treated with LRP versus RRP. They reported similar potency rates 1 year after surgery favoring the LRP group on account of more frequent spontaneous erections. This was attributed to better visualization and consequently better nerve sparing during LRP [19]. Hara et al. [32] also compared the two technical approaches. They used two questionnaires: the EORTC QLQ-C30 to estimate HRQOL in general, and the IIEF-5 to estimate sexual quality of life. Sexual life was significantly impaired after the operation independent of the approach, but after LPR men were more satisfied in general. However, their study was performed in a cross-sectional way using data collected at 6 months or later after surgery [32].

In order to satisfactorily preserve the sexual function of patients after LRP, careful preservation of the neuro- vascular bundles is needed. Sexual dysfunction seems to be an important complication of LRP that generates significant discomfort to men and their sexual partners.

\section{Bowel Function}

Bowel dysfunction may occur after RP and it is marked by fecal incontinence (FI). FI occurs due to the loss of control of the anal sphincter. Consequently, these patients are unable to control the release of colon content. This inability dramatically influences self-confidence, social life and HRQOL in general. Age is a significant predisposing factor for FI. In elderly people, FI happens in up to $4 \%$. Continence of stool is the result of a complex mechanism which includes the integrity of the anal sphincter and the rectum, and it is influenced by stool volume and consistency. During an operation in the region of these anatomical structures, injury may occur, leading to various degrees of FI.

FI is a complication which seems to happen more often when performing a perineal RP. Bishoff et al. [33] showed a higher prevalence of less than monthly FI in men after the perineal versus retropubic approach. Moreover, after perineal RP, patients were more likely to wear pads for stool leakage, to have a larger amount of stool leakage and to experience more events compared to the retropubic group [33]. Recently, Namiki et al. [23] showed no significant difference in bowel complication rates in men with retropubic, perineal or laparoscopic RP.

It is difficult to estimate the presence of bowel dysfunction before and after RP. The questionnaires used to date in different published series are just instruments for abundant identification of men with post-prostatectomy alterations in bowel function. Afterwards, a careful medical history, physical examination and further laboratory examination are needed [9].

\section{Conclusions}

$\mathrm{RP}$ is a standard approach for the management of prostate cancer. As the oncological outcome has improved, focus has been drawn to the amelioration of postoperative HRQOL. Urinary and sexual dysfunction as well as FI form the three major complications after surgery which may affect a patient's quality of life. In this concept, meticulous counseling regarding the potential morbidity associated with the operation that may affect the patient's quality of life should be made before obtain- 
ing informed consent. Partners should also be present at this counseling because they are indirectly or directly affected by the diagnosis and the subsequent possible functional disorders after treatment. Questionnaires only partially help to identify patients with dysfunction, especially sexual and bowel dysfunction, and careful patient counseling is the best way to evaluate the presence of a dysfunction pre- or postoperatively.

\section{References}

1 American Cancer Society: Cancer Facts and Figures 2003. http://www.cancer.org/ downloads/STT/CAFF2003PWSecured.pdf. Accessed June 23, 2006.

-2 Schuessler WW, Schulam PG, Clayman RV, et al: Laparoscopic radical prostatectomy: initial short-term experience. Urology 1997; 50:854-857.

3 Rassweiler J, Schulze M, Teber D, et al: Laparoscopic radical prostatectomy: functional and oncological outcomes. Curr Opin Urol 2004; 14:75-82.

-4 Stolzenburg JU, Truss MC: Technique of laparoscopic (endoscopic) radical prostatectomy. BJU Int 2003;91:749-757.

5 Stolzenburg JU, Truss MC, Do M, et al: Evolution of endoscopic extraperitoneal radical prostatectomy (EERPE) - technical improvements and development of a nervesparing, potency-preserving approach. World J Urol 2003;21:147-152.

-6 Stolzenburg JU, Do M, Rabenalt R, et al: Endoscopic extraperitoneal radical prostatectomy (EERPE) - initial experience after 70 procedures. J Urol 2003;169:2066-2071.

7 Guillonneau B, Vallancien G: Laparoscopic radical prostatectomy: initial experience and preliminary assessment after 65 operations. Prostate 1999;39:71-75.

-8 Salomon L, Levrel O, de la Taille, et al: Radical prostatectomy by the retropubic, perineal and laparoscopic approach: 12 years of experience in one center. Eur Urol 2002;42:104110.

-9 Kirschner-Hermanns R, Jakse G: Quality of life following radical prostatectomy. Crit Rev Oncol Hematol 2002;43:141-151.

10 Schipper H, Clinch J, McMurray A, et al: Measuring the quality of life of cancer patients: the Functional Living Index-Cancer: development and validation. J Clin Oncol 1984;2:472-483.

-11 Aaronson NK, Ahmedzai S, Bergman B, et al: The European Organization for Research and Treatment of Cancer QLQ-C30: A quality-of-life instrument for use in international clinical trials in oncology. J Natl Cancer Inst 1993;85:365-376.

12 Kelleher CJ, Cardozo LD, Khullar V, et al: A new questionnaire to assess the quality of life of urinary incontinent women. Br J Obstet Gynaecol 1997;104:1374-1379.
13 Wagner TH, Patrick DL, Bavendam TG, et al: Quality of life of persons with urinary incontinence: development of a new measure. Urology 1996;47:67-71.

14 Jackson S, Donovan J, Brookes S, et al: The Bristol Female Lower Urinary Tract Symptoms questionnaire: development and psychometric testing. Br J Urol 1996;77:805812.

15 Lee PS, Reid DW, Saltmarche A, et al: Measuring the psychosocial impact of urinary incontinence: the York Incontinence Perceptions Scale (YIPS). J Am Geriatr Soc 1995; 43:1275-1278.

16 Shumaker SA, Wyman JF, Uebersax JS, et al: Health-related quality of life measures for women with urinary incontinence: the Incontinence Impact Questionnaire and the Urogenital Distress Inventory: Continence Program in Women (CRW) Research Group Qual Life Res 1994;3:291-306.

17 Moore K, Jensen L: Testing of the Incontinence Impact Questionnaire (IIQ-7) with men after radical prostatectomy. J Wound Ostomy Continence Nurs 2000;27:304-312.

Ostomy Continence Nurs 2000;27:304-312.
Namiki S, Egawa S, Baba S, et al: Recovery of quality of life in year after laparoscopic or retropubic radical prostatectomy: a multiinstitutional longitudinal study. Urology 2005;65:517-523.

$\checkmark 19$ Roumeguere T, Bollens R, Vanden Bossche $\mathrm{M}$, et al: Radical prostatectomy: a prospective comparison of oncological and functional results between open and laparoscopic approaches. World J Urol 2003;20: 360-366.

20 Anastasiadis AG, Salomon L, Katz R, et al: Radical retropubic versus laparoscopic prostatectomy: a prospective comparison of functional outcome. Urology 2003;62:292297.

21 Rassweiler J, Seemann O, Schulze M, et al: Laparoscopic versus open radical prostatectomy: a comparative study at a single institution. J Urol 2003;169:1689-1693.

22 Eden CG, Cahill D, Vass JA, et al: Laparoscopic radical prostatectomy: the initial UK series. BJU Int 2002;90:876-882.
23 Namiki S, Egawa S, Baba S, et al: Changes in quality of life in first year after radical prostatectomy by retropubic, laparoscopic, and perineal approach: multi-institutional longitudinal study in Japan. Urology 2006;67: 321-327.

24 Helgason AR, Adolfson J, Dickman P, et al: Waning sexual function - the most important disease-specific distress for patients with prostate cancer. Br J Cancer 1996;73: 1417-1421.

25 Schag CA, Heinrich RL: Developments of a comprehensive quality of life measurement tool: CARES. Oncology (Williston Park) 1990;4:135-138.

26 Ganz PA, Schag CA, Lee JJ, et al: The CARES: a generic measure of health-related quality of life for patients with cancer. Qual Life Res 1992;1:19-29.

27 O'Leary MP, Fowler FJ, Lenderking WR, et al: A brief male sexual function inventory for urology. Urology 1995;46:697-706.

28 Shrader-Bogen CL, Kjellberg JL, McPherson $\mathrm{CP}$, et al: Quality of life and treatment outcomes: prostate carcinoma patients' perspectives after prostatectomy or radiation therapy. Cancer 1997;79:1977-1986.

29 Wei JT, Dunn RL, Litwin MS, et al: Development and validation of the expanded prostate cancer index composite (EPIC) for comprehensive assessment of health-related quality of life in men with prostate cancer. Urology 2000;56:899-905.

30 Wagner A, Link R, Pavlovich C, et al: Use of a validated quality of life questionnaire to assess sexual function following laparoscopic radical prostatectomy. Int J Impot Res 2006; 18:69-76.

31 Katz R, Salomon L, Hoznek A, et al: Patient reported sexual function following laparoscopic radical prostatectomy. J Urol 2002; 168:2078-2082.

32 Hara I, Kawabata G, Miyake H, Nakamura I, Hara S, Okada H, Kamidono S: Comparison of quality of life following laparoscopic and open prostatectomy for prostate cancer. J Urol 2003;169:2045-2048.

>33 Bishoff JT, Motley G, Optenberg SA, et al: Incidence of fecal and urinary incontinence following radical perineal and retropubic prostatectomy in a national population. J Urol 1998;160:454-458. 